\title{
Combination therapies for the management of nocturia and its comorbidities
}

This article was published in the following Dove Press journal:

Research and Reports in Urology

20 April 2015

Number of times this article has been viewed

\section{Cenk Murat Yazici \\ Omer Kurt}

Department of Urology, Namik Kemal University School of Medicine, Tekirdag, Turkey
Correspondence: Cenk Murat Yazici Department of Urology, Namik Kemal University School of Medicine, 100. YIl Mahallesi, Tunca Caddesi, 59100 Merkez/ Tekirdağ, Turkey

Tel +90506 8552687

Fax +90282 250500 I

Email drcenkyazici@yahoo.com

\begin{abstract}
Nocturia is the most bothersome lower urinary tract symptom. It has a multifactorial etiology. It had been thought nocturia was a nonspecific symptom of lower urinary system dysfunction, but it has been determined that many diseases, related to different organ systems, might be reasons for this nonspecific symptom. Along with the importance of systemic diseases that cause nocturia, the symptom itself has adverse effects on patients' health and quality of life. There are several studies reporting a direct relationship between nocturia and depression, cognitive dysfunction, mood disturbances, falls, and fractures. For this reason, it is important to treat nocturia both to increase quality of life and to decrease related complications. Treatment opportunities have been under investigation for 20 years. Most of the studies in the literature have reported the results of single-drug medication on nocturia, which may be insufficient for a situation that has such a multifactorial etiology. In this review, we evaluated the success of different treatment combinations on nocturia.
\end{abstract}

Keywords: nocturia, combination, treatment, polyuria, lower urinary tract symptoms

\section{Introduction}

Nocturia has become one of the most popular topics in urology in the last decade. It was thought to be a simple lower urinary tract symptom, but recent studies have confirmed the significance of this condition with regard to human health. The International Continence Society has defined nocturia as a complaint wherein the individual must wake up one or more times at night to void, independent of age, sex, causes, and associated bother. The main point of this definition was that nocturnal voids must precede and be followed by sleep. ${ }^{1,2}$ According to this definition, first morning voids should not be accepted as nocturia. This also distinguishes nocturia from nighttime frequency, defined as a number of night voids that are not related with sleep. ${ }^{2}$ Another terminological entity that should be distinguished from nocturia is nocturnal enuresis. In nocturia, patients are aware of having a full bladder and wake up to void, which is not the situation in nocturnal enuresis. Patients with nocturnal enuresis are not aware of their full bladders, and thus involuntarily empty their bladder into their beds. These terminological differences are key points for clinicians in differentiating several forms of nighttime voiding dysfunction.

There is still no consensus about the frequency of nocturia. Although the International Continence Society classification used the term "one or more times," some authors have reported the frequency of nocturia to be two or more episodes/night. For this reason, there have been different prevalence rates of nocturia in literature. In a recent meta-analysis, the prevalence of nocturia, which was defined as two or more 
episodes/night, has been reported as $2 \%-18 \%$ in the patients aged $20-40$ years, increasing to $28 \%-62 \%$ by age 70 years. ${ }^{3}$ Nocturia occurring at least once a night was reported to be more prevalent in younger women than younger men, but the prevalence became similar in both sexes in older age groups. ${ }^{4,5}$ Regardless of the different definitions, there are two facts about the epidemiological properties of nocturia that remain constant: it is a common situation, and the prevalence rates increase with age. This age-dependent relationship has been shown in several epidemiological studies about nocturia. ${ }^{3-5}$

\section{Risk factors and etiology of nocturia}

There are several etiological factors for nocturia, but the main pathophysiological mechanisms are related to four main issues: nocturnal polyuria, global polyuria, diminished nocturnal bladder capacity, and sleep impairment (Table 1). Different organ systems may take part in the formation of nocturia. Diseases related to the cardiovascular system (congestive heart disease), respiratory system (obstructive sleep apnea), urogenital system (renal dysfunction, detrusor overactivity, neurogenic bladder, etc), and psychosomatic

Table I The pathophysiological factors and etiology of nocturia
I. Nocturnal polyuria
a. Decrease in nocturnal vasopressin
b. Increase in atrial natriuretic peptide
c. Cardiac insufficiency, congestive heart failure
d. Obstructive sleep apnea
e. Diuretic usage (at evening)
f. Lower extremity venous insufficiency
g. Evening polydipsia, alcoholism

2. Global polyuria

a. Diabetes insipidus

b. Diabetes mellitus

c. Estrogen insufficiency in women

d. Habitual polydipsia

e. Renal insufficiency

f. Hypercalcemia- and hypercalciuria-related diseases

3. Decreased nocturnal bladder capacity

a. Detrusor overactivity

b. Bladder outlet obstruction and related postvoidal residual urine

c. Bladder hypersensitivity

d. Urinary tract infection

e. Bladder wall fibrosis

f. Bladder tumor, stone, or foreign body

g. Interstitial cystitis

h. Postsurgical bladder dysfunction

4. Sleep disturbances

a. Environmental impairments

b. Anxiety disorders

c. Depression

d. Stimulant usage

e. Melatonin deficiency of aging system (anxiety disorders, depression) may be a reason for nocturia. Patients' medical treatments for their comorbities, and even insomnia, may also cause nocturia. This etiological complexity complicates the evaluation and treatment of nocturia. Another important issue for nocturia is that patients may have overlapping causative factors. For those patients, evaluation is an extremely challenging situation and needs a multidisciplinary workshop.

Nocturnal polyuria is the most frequent cause of nocturia, defined as nighttime urine output higher than $20 \%$ of total daily urine output for younger adults and higher than 33\% for older adults. ${ }^{2}$ It was shown that nearly $80 \%$ of patients with nocturia had nocturnal polyuria. ${ }^{6}$ It may be behavioral, similar to excess fluid intake; drug-induced; or a part of global polyuria. Systemic diseases such as congestive heart disease, venous stasis, obstructive sleep apnea, renal tubular dysfunction, hepatic failure, and hypoalbuminemia all can cause fluid and electrolyte sequestration and may be a reason for nocturnal polyuria. Global polyuria is defined as a daily urine volume greater than $40 \mathrm{~mL} / \mathrm{kg}$ and can be seen in patients with diabetes insipidus, diabetes mellitus, excessive fluid intake, hypercalcemia, or primary polydipsia, or it can be drug-induced. ${ }^{7}$ Lower urinary tract dysfunctions related to storage or voiding pathologies may also cause nocturia, affecting the functional capacity or somatosensory properties of the bladder. Reduced nocturnal bladder capacity can occur with high residual urine levels, bladder pain syndrome, dysfunctional voiding, intravesicular masses or stones, pelvic organ prolapses, or the external compression of bladder, or it can be drug-induced. These patients are not able to store the amount of urine produced at night and must void. Regardless of any physiological abnormality, psychosomatic disorders, various medications, and sleep disturbances can also be a reason for nocturia, and they must be checked during nocturia evaluation. ${ }^{7}$

\section{Clinical aspects of nocturia}

Etiological factors for nocturia may vary by age. It was shown that decreased nocturnal bladder capacity was the main pathology for the younger population, whereas nocturnal polyuria was more prevalent at older ages. ${ }^{8}$ Nocturnal polyuria may not be related to daytime symptoms, and nocturia may be the only manifestation of several systemic diseases. For this reason, evaluation of nocturia has vital importance in diagnosing a systemic disease. In addition, nocturia may be a bothersome situation. Several studies have reported nocturia to be one of the most bothersome lower urinary system symptoms. ${ }^{8}$ Although the perception of bother is a 
relative situation, most studies showed that two voids per night is the threshold for nocturia to be bothersome and significantly decrease quality of life..$^{7-9}$ Nocturia can also cause both psychological and physiological damage. The prevalences of depression, cognitive dysfunction, mood disturbances, falls, and fractures were reported to be higher in patients with nocturia. ${ }^{8-11}$

One of the most useful diagnostic tools for nocturia is a frequency-volume chart. It is a cheap and noninvasive diagnostic method that can give important information about the patient. With a simple 24-72 hour voiding diary, clinicians may estimate bladder capacity, voiding and drinking habits, and daily/nighttime urine production and may detect incontinence episodes. These data are helpful in distinguishing nocturnal polyuria, global polyuria, and reduced bladder capacity and guide the clinicians in choosing additional diagnostic tests to identify the underlying pathology. The frequency-volume chart is also important for the follow-up of treatment success and can be classified as "mandatory" for the evaluation of nocturia. Another easy and cheap diagnostic tool is the quality-of-life questionnaire, which can detect the bothersome effect of nocturia. Several validated questionnaires have been used, and the Nocturia-Quality of Life questionnaire may be suitable for this evaluation. ${ }^{12,13}$

\section{Management of nocturia}

Nocturia is a nonspecific situation that may relatively affect the quality of life. For this reason, an individualized treatment model must be planned. To plan the exact treatment strategy, appropriate evaluation of nocturia and documentation of risk factors are the main points. Patients and clinicians must understand that nocturia is a multifactorial situation. In some patients, multiple treatment options must be combined to treat a single symptom. In contrast, it may sometimes be impossible to treat nocturia completely, as it may be resistant to proper treatments. Patients must be informed about the goals of treatment, which are to decrease nocturia episodes, to increase the quality of life, to increase total sleep time, and to diminish associated comorbities. Several treatment opportunities are used to treat nocturia. These include behavioral interventions, $\alpha$-blockers, 5- $\alpha$ reductase inhibitors, antimuscarinics, serotonin reuptake inhibitors, desmopressin, diuretics, and a combination of these treatments. ${ }^{14}$

Behavioral interventions are an easy, cheap, and effective form of treatment. These include restriction of nighttime fluid, avoiding nighttime caffeinated and alcoholic beverages, voiding before going to sleep, offering regular physical activity, and leg elevation for patients with peripheral edema. There are very limited numbers of randomized prospective controlled studies evaluating the effect of behavioral interventions alone or in combination with medical treatments. In a prospective study, Soda et al detected more than 50\% improvement of nocturia with behavioral interventions. ${ }^{15}$ In another study with a limited number of overactive bladder patients, reducing the fluid intake by $25 \%$ statistically decreased nocturia episodes, but this decrease was not clinically significant (mean, 1.4 to 1.3 episodes/night). When the patients decreased their fluid intake by $32 \%$, nocturia episodes decreased from 2.3 episodes/night to 1.8 episodes/night. ${ }^{16}$ In a 4-week uncontrolled study, with the combination of behavioral intervention, terazosin, and zaleplon, mean nocturia episodes were reduced from 2.6 episodes/night to 1.9 episodes/night. ${ }^{17}$ Although there has been a relative paucity of supporting data related to the combination of behavioral interventions with medical treatment, the panelists of New England Research Institutes Inc. concluded that "multicomponent interventions are [an] attractive approach for nocturia." 18

Although nocturia is one of the most prevalent symptoms of benign prostate hyperplasia $(\mathrm{BPH})$, there are limited numbers of studies evaluating the effect of BPH treatment, especially on nocturia. Several drugs, such as $\alpha$-blockers, $5-\alpha$ reductase inhibitors, an $\alpha$-blocker and 5- $\alpha$ reductase inhibitor combination, phosphodiesterase inhibitors, and phytotherapeutic agents, were used. Oelke et al analyzed these studies in their review article. ${ }^{8}$ The authors reported that nearly half of the patients had improvement in nocturia with these medications, but the mean improvement rates were between $9.5 \%$ and $33 \%$. Although improvement of nocturia was significantly better with medical treatments, there were a considerable number of patients who also responded to placebo treatment. The reduction of nocturia with medical treatment was modest compared with placebo, and the difference was nearly 0.2 voids/night. ${ }^{8}$ A subset analysis of the Medical Therapy of Prostatic Symptoms study at the first year of treatment showed that a combination of doxazosin and finasteride improved nocturia frequency significantly compared with placebo. However, improvement of nocturia was also the same in the doxazosin group, which means combining 5- $\alpha$ reductase inhibitors and $\alpha$-blockers did not increase the effect of monotherapy. ${ }^{19}$ Similar findings were also observed in the COMBAT-study: that a combination of tamsulosin and dutasteride did not make any clinically significant difference for the treatment of nocturia. ${ }^{20}$ In the Veterans' Administration Cooperative Study, terazosin, finasteride, and a combination of these drugs were used and compared with placebo. The improvement of nocturia was 
a shift from 2.4 to 1.8 episodes/night with terazosin, 2.5 to 2.1 episodes/night with finasteride, 2.4 to 2.0 episodes/night with the combination, and 2.4 to 2.1 episodes/night in the placebo group. Although the reduction of nocturia was significant in the treatment group, the clinical significance of these results was debatable. ${ }^{21}$ In another study, 5- $\alpha$ receptor inhibitors did not show any benefit for nocturia, and they did not have any additive effect on $\alpha$-blocker treatment. ${ }^{22}$ As a result, $\alpha$-blocker treatment was seen to have a minimal effect on nocturia of patients with $\mathrm{BPH}$, but a combination of 5- $\alpha$ reductase inhibitors with $\alpha$-blockers did not increase the efficacy of treatment. Desmopressin treatment alone or in combination is another option for BPH patients with nocturnal polyuria. In a study from Germany, it was shown that desmopressin treatment significantly decreased nocturia frequency and improved the quality of life. The authors reported a decrease in nocturia frequency by $53 \%$ (from 3.8 to 1.7 episodes/night) with desmopressin monotherapy, but the combination of desmopressin and an $\alpha$-blocker did not show any synergistic effect. ${ }^{23}$ The combination effect of $\alpha$-blockers and phosphodiesterase 5 inhibitors on nocturia has been studied by Kaplan et al. ${ }^{24}$ There was a significant improvement on IPSS scores of patients in both alfuzosin monotherapy and combination treatment, but the effect of these treatments on nocturia had not been evaluated separately. ${ }^{24}$

Overactive bladder is another common cause of nocturia. Antimuscarinic medication is the first-line treatment for those patients. There are some controversies about the efficacy of antimuscarinic treatment on nocturia. In a double-blind placebo controlled study with solifenacin 5 and $10 \mathrm{mg}$, a significant reduction in nocturia frequency was observed, but the clinical significance of this reduction was debatable (reduction, 0.71 episodes/night with the drug and 0.52 episodes/night with the placebo). ${ }^{25}$ Similar reduction rates were observed in the Solifenacin and Tolterodine as an Active Comparator randomized trial. The frequency of nocturia decreased 0.71 episodes/night with solifenacin and 0.63 episodes/night with tolterodine, but there was no placebo group. ${ }^{26}$ In another study, fesoterodine showed a statistically significant but clinically insignificant reduction in nocturia frequency. ${ }^{27}$ The efficacy of antimuscarinics on nocturia was supposed to be related, with an underlying pathology. Brubaker et al reported that $62 \%$ of their patients with overactive bladder also had nocturnal polyuria, and the treatment success of these patients with antimuscarinic medication was less than for patients without nocturnal polyuria. ${ }^{28}$ These data showed that antimuscarinic treatment was more effective in nocturia if the patient had overactive bladder as the causative factor. A combination of antimuscarinic and antidiuretic treatment may be a feasible option for those patients who also have nocturnal polyuria. The combination treatment with $\alpha$-blockers and antimuscarinics had also been studied. In a study combining tamsulosin with tolterodine for the treatment of patients with BPH and overactive bladder, nocturia episodes were significantly decreased, but the difference between treatment and placebo group was only 0.2 episodes/ night. ${ }^{29}$ In another study evaluating the effect of a tamsulosin and solifenacin combination on nocturia, the authors reported no significant difference of combination treatment compared with either monotherapy with tamsulosin or solifenacin. ${ }^{30}$ As a result, the combination of antimuscarinics and the $\alpha$-blockers was seen to be a feasible solution for nocturia, but there was no study demonstrating the additive effect of this treatment. The panelists of the New England Research Institutes Inc. concluded that antimuscarinic treatment for nocturia was minimally more effective than placebo. This treatment can be more effective in patients with overactive bladder and as a part of multimodality treatment for complex lower urinary system dysfunction. ${ }^{18}$

Antidiuretic therapy with desmopressin is the only treatment that has a grade A recommendation and was approved in some countries for the treatment of nocturia. ${ }^{31,32}$ It is a synthetic analog of vasopressin, increasing water absorption from the distal collecting tubule by increasing the action of V2 receptors. Desmopressin has been used for diabetes insipidus and nocturnal enuresis for more than 30 years. Because nocturnal polyuria has been defined as an etiological factor for nocturia, desmopressin treatment was shown to be useful for the treatment of nocturia. It has been scientifically proven that women have higher sensitivity to desmopressin treatment than men, so higher dosages had to be given to male patients to maintain the same effect as women. ${ }^{33}$ Antidiuretic treatment with desmopressin was able to reduce nocturia episodes more than $50 \%$, which was a significant response compared with placebo. ${ }^{34}$

In a randomized study, nearly $35 \%$ of patients had more than $50 \%$ reduction in nocturia episodes with desmopressin; the decrease was only $10 \%$ in a placebo group. ${ }^{35}$ In terms of quality of life, antidiuretic treatment was able to increase initial sleep time, and patients reported less fatigue in the corresponding day. ${ }^{34}$ The number of patients who reported nocturia as a bothersome situation decreased with desmopressin treatment. ${ }^{36}$ Antidiuretic treatment was more effective in patients with severe nocturnal polyuria. In a study of patients with three or more nocturia episodes/night, desmopressin treatment decreased the mean nocturnal urine volume 
from $955 \pm 255$ to $522 \pm 210 \mathrm{~mL}$. The mean nocturnal voiding episodes also decreased from $5.20 \pm 1.16$ to $2.24 \pm 1.12$ $\mathrm{mL} .{ }^{37}$ Desmopressin was well tolerated, and the adverse events were similar to those of the placebo group. The main problem for such treatment in elderly patients was the risk for hyponatremia. Hyponatremia was dose- and age-related and was generally seen in the first week of treatment. It has been agreed that desmopressin must not be given to patients with polyuria of unknown etiology and with hyponatremia. ${ }^{14}$ It was recommended that serum sodium level be assessed at the third day and the first week of treatment, so as to not underestimate asymptomatic hyponatremia. Excessive fluid intake should be avoided after desmopressin treatment, and if there is a sign of fluid retention such as leg edema, treatment should be discontinued. Nocturnal polyuria may be seen with solute or nonsolute diuresis; it is expected that antidiuretic treatment will be more successful in nonsolute diuresis patients. Urine osmolarity can be used as an important marker to estimate the success of desmopressin treatment in patients with nocturnal polyuria. These data are still theoretical and need to be proved by scientific studies.

Diuretic treatment has a paradoxical effect, in that it can both induce and decrease nocturia. The timing of diuretic treatment is the main predictor for this paradoxical effect. The aim of this treatment for nocturia is to induce diuresis before sleep and to shift the polyuria phase from sleep to daily life. For this purpose, diuretics should be given in the midafternoon. Administration of these drugs during nighttime would exacerbate nocturnal polyuria and might increase nocturia episodes. It was shown that $40 \mathrm{mg}$ furosemide 6 hours before sleep was able to decrease nocturia frequency without decreasing the nocturnal voided volume. ${ }^{38}$ Diuretic treatment with bumetanide and furosemide was confirmed by the Committee for Establishment of the Clinical Guidelines for Nocturia of the Neurogenic Bladder Society as a treatment opportunity with a grade $\mathrm{C}$ recommendation. ${ }^{39}$ There are very limited data about diuretic treatment on nocturia in combination with other drugs. In their study, Cho et al evaluated the efficacy of the hydrochlorothiazide plus terazosin combination on 53 patients with nocturia. They reported that nearly $30 \%$ of their patients had a more than $50 \%$ reduction in nocturia frequency, but there was no placebo group in their study. In the light of this information, it is impossible to make a direct conclusion about the efficacy of diuretic combinations on treatment of nocturia; more randomized studies are needed.

Nocturia can be seen in patients with sleep disturbances. Many physical (chronic pain, chronic cough, nighttime heartburn, etc) and psychological (depression, mood disorders, anxiety, etc) diseases can be a reason for sleep impairment. Improving the sleep environment and decreasing disturbing stimuli such as noise and light eventually provided a comfortable sleep for patients and increased their quality of life. ${ }^{41}$ Treating the underlying pathology that causes sleep disturbances such as chronic cough, chronic pain, heartburn, and so on may concurrently decrease nocturia episodes. Patients with sleep disturbances must be advised to have relaxation therapies, which can be a solution for insomniacs. Specific medications that may cause nocturnal polyuria or nocturnal voiding problems must be rescheduled to decrease their unwanted effects during the night.

There is a significant relationship between depression and nocturia. It was shown that nocturia increased the odds of reporting depression (mean 1.2-20.2), whereas depression also increased the odds of reporting the outcome of nocturia (mean 1.2-7.7). ${ }^{42}$ Although there are some studies reporting that serotonin reuptake inhibitors double the risk for nocturia, there are not enough scientific data indicating that antidepressants or antipsychotic medication cause nocturia. ${ }^{43}$ In contrast, nocturia and depression are frequently comorbid situations, and clinicians must simply evaluate the presence of depression in patients with nocturia. These patients must be referred to a specialist for treatment. Obstructive sleep apnea can also be a particular reason for nocturia. The main mechanism for nocturia in obstructive sleep apnea is related to pulmonary vasoconstriction, which stimulates the release of atrial natriuretic peptide from the atria. This hormone induces renal sodium and water excretion, which in turn causes nocturia. The treatment of this situation with continuous positive airway pressure was effective in reducing nocturia frequency from 2.6 to 0.7 episodes/night. ${ }^{44}$

Nonsteroidal anti-inflammatory drugs were intended to decrease the glomerular filtration rate, which was thought to be a solution for nocturnal polyuria. In a small study with 26 patients, diclofenac reduced nocturia frequency by $18 \%$, and the difference between study and the placebo group was significant. In contrast, the placebo response in this study was much lower than usual (3.5\% decrease) ${ }^{45}$ In another study with naproxen, a significant decrease in nocturia frequency ( -0.7 episodes/night) was observed. ${ }^{46}$ There are still not enough data to prove the efficacy of nonsteroidal anti-inflammatory drugs on nocturia treatment. Melatonin was also used for nocturia treatment. The aim of this treatment is to restore a regular daily sleep-wake rhythm. There are a limited number of studies about this treatment, and the results are conflicting. In one study, melatonin was able to 
decrease nocturia episodes a mean of 0.32 per night, which was significantly higher than placebo. However, the mean decrease of nocturia episodes in the placebo group of this study was much lower ( 0.05 episodes/night) than in other related studies. There was no significant decrease in nocturnal urine volume with melatonin. ${ }^{47}$

\section{Conclusion and recommendations}

Nocturia is a highly prevalent and bothersome symptom of the lower urinary tract. Several diseases related to different organ systems may cause nocturia. It may result in both physiological and psychological damage and even may increase the mortality of patients. Clinicians must be aware of this situation and understand the complexity of the evaluation needed to offer the exact treatment modality for nocturia. Most of the studies in the literature reported the statistical difference of response to various treatments, but the main point for these patients is clinical significance. Quality-of-life changes of patients must also be evaluated to define the real success for treatments of nocturia, which actually matter to the patients. In addition to this, evaluating the effect of treatment on the complication of nocturia, such as falls and fractures, must be the secondary aim of such studies. These data will be able to show the exact success of treatment for nocturia.

Nocturia is a complex situation with several responsible pathophysiological mechanisms. Different etiological factors may take place in the same patient. An individualized model of treatment is the best option for nocturia treatment. For this reason, patients must be evaluated for all possible etiological factors and must be treated according to this evaluation. The complex nature of nocturia may need a combination treatment that can cover multiple etiological factors. Although this treatment modality seems to be logical, there are a limited number of studies evaluating combination treatments in nocturia. Future studies focusing on this subject may determine the success of combination treatments on nocturia.

\section{Disclosure}

The authors report no conflicts of interest in this work.

\section{References}

1. Abrams P, Cardozo L, Fall M, et al; Standardisation Sub-committee of the International Continence Society. The standardisation of terminology of lower urinary tract function: report from the Standardisation Subcommittee of the International Continence Society. Neurourol Urodyn. 2002;21(2):167-178.

2. van Kerrebroeck P, Abrams P, Chaikin D, et al; Standardisation Subcommittee of the International Continence Society. The standardisation of terminology in nocturia: report from the Standardisation Sub-committee of the International Continence Society. Neurourol Urodyn. 2002;21(2): 179-183.
3. Bosch JL, Weiss JP. The prevalence and causes of nocturia. $J$ Urol. 2010;184(2):440-446.

4. McGrother CW, Donaldson MM, Shaw C, et al; MRC Incontinence Study Team. Storage symptoms of the bladder: prevalence, incidence and need for services in the UK. BJU Int. 2004;93(6):763-769.

5. Tikkinen KA, Tammela TL, Huhtala H, Auvinen A. Is nocturia equally common among men and women? A population based study in Finland. J Urol. 2006;175(2):596-600.

6. Chang SC, Lin AT, Chen KK, Chang LS. Multifactorial nature of male nocturia. Urology. 2006;67(3):541-544.

7. Cornu JN, Abrams P, Chapple CR, et al. A contemporary assessment of nocturia: definition, epidemiology, pathophysiology, and management - a systematic review and meta-analysis. Eur Urol. 2012;62(5):877-890.

8. Oelke M, Adler E, Marschall-Kehrel D, Herrmann TR, Berges R. Nocturia: state of the art and critical analysis of current assessment and treatment strategies. World J Urol. 2014;32(5):1109-1117.

9. Oelke M, Wiese B, Berges R. Nocturia and its impact on healthrelated quality of life and health care seeking behaviour in German community-dwelling men aged 50 years or older. World J Urol. 2014;32(5):1155-1162.

10. Vaughan CP, Brown CJ, Goode PS, Burgio KL, Allman RM, Johnson TM II. The association of nocturia with incident falls in an elderly community-dwelling cohort. Int J Clin Pract. 2010;64(5):577-583.

11. Asplund R. Hip fractures, nocturia, and nocturnal polyuria in the elderly. Arch Gerontol Geriatr. 2006;43(3):319-326.

12. Weiss JP, Bosch JL, Drake M, et al. Nocturia Think Tank: focus on nocturnal polyuria: ICI-RS 2011. Neurourol Urodyn. 2012;31(3): 330-339.

13. Abraham L, Hareendran A, Mills IW, et al. Development and validation of a quality-of-life measure for men with nocturia. Urology. 2004;63(3): 481-486.

14. Weiss JP, Wein AJ, van Kerrebroeck P, et al. Nocturia: new directions. Neurourol Urodyn. 2011;30(5):700-703.

15. Soda T, Masui K, Okuno H, Terai A, Ogawa O, Yoshimura K. Efficacy of nondrug lifestyle measures for the treatment of nocturia. $J$ Urol. 2010;184(3):1000-1004.

16. Hashim H, Abrams P. How should patients with an overactive bladder manipulate their fluid intake? BJU Int. 2008;102(1):62-66.

17. Vaughan CP, Endeshaw Y, Nagamia Z, Ouslander JG, Johnson TM. A multicomponent behavioural and drug intervention for nocturia in elderly men: rationale and pilot results. BJU Int. 2009;104(1):69-74.

18. Weiss JP, Blaivas JG, Blanker MH, et al. The New England Research Institutes, Inc. (NERI) Nocturia Advisory Conference 2012: focus on outcomes of therapy. BJU Int. 2013;111(5):700-716.

19. Johnson TM II, Burrows PK, Kusek JW, et al; Medical Therapy of Prostatic Symptoms Research Group. The effect of doxazosin, finasteride and combination therapy on nocturia in men with benign prostatic hyperplasia. J Urol. 2007;178(5):2045-2050.

20. Montorsi F, Roehrborn C, Garcia-Penit J, et al. The effects of dutasteride or tamsulosin alone and in combination on storage and voiding symptoms in men with lower urinary tract symptoms (LUTS) and benign prostatic hyperplasia (BPH): 4-year data from the Combination of Avodart and Tamsulosin (CombAT) study. BJU Int. 2011;107(9):1426-1431.

21. Johnson TM II, Jones K, Williford WO, Kutner MH, Issa MM, Lepor $\mathrm{H}$. Changes in nocturia from medical treatment of benign prostatic hyperplasia: secondary analysis of the Department of Veterans Affairs Cooperative Study Trial. J Urol. 2003;170(1):145-148.

22. Weiss JP, Blaivas JG, Bliwise DL, et al. The evaluation and treatment of nocturia: a consensus statement. BJU Int. 2011;108(1):6-21.

23. Berges R, Höfner K, Gedamke M, Oelke M. Impact of desmopressin on nocturia due to nocturnal polyuria in men with lower urinary tract symptoms suggestive of benign prostatic hyperplasia (LUTS/ BPH). World J Urol. 2014;32(5):1163-1170.

24. Kaplan SA, Gonzalez RR, Te AE. Combination of alfuzosin and sildenafil is superior to monotherapy in treating lower urinary tract symptoms and erectile dysfunction. Eur Urol. 2007;51(6):1717-1723. 
25. Cardozo L, Lisec M, Millard R, et al. Randomized, double-blind placebo controlled trial of the once daily antimuscarinic agent solifenacin succinate in patients with overactive bladder. $J$ Urol. 2004;172(5 Pt 1): 1919-1924.

26. Chapple CR, Martinez-Garcia R, Selvaggi L, et al; STAR study group. A comparison of the efficacy and tolerability of solifenacin succinate and extended release tolterodine at treating overactive bladder syndrome: results of the STAR trial. Eur Urol. 2005;48(3):464-470.

27. Chapple C, Van Kerrebroeck P, Tubaro A, et al. Clinical efficacy, safety, and tolerability of once-daily fesoterodine in subjects with overactive bladder. Eur Urol. 2007;52(4):1204-1212.

28. Brubaker L, FitzGerald MP. Nocturnal polyuria and nocturia relief in patients treated with solifenacin for overactive bladder symptoms. Int Urogynecol J Pelvic Floor Dysfunct. 2007;18(7):737-741.

29. Kaplan SA, Roehrborn CG, Rovner ES, Carlsson M, Bavendam T, Guan Z. Tolterodine and tamsulosin for treatment of men with lower urinary tract symptoms and overactive bladder: a randomized controlled trial. JAMA. 2006;296(19):2319-2328.

30. Yamaguchi O, Kakizaki H, Homma Y, et al; ASSIST Study Group. Solifenacin as add-on therapy for overactive bladder symptoms in men treated for lower urinary tract symptoms - ASSIST, randomized controlled study. Urology. 2011;78(1):126-133.

31. Andersson KE. The pharmacological treatment of nocturia. BJU Int. 2002;90(Suppl 3):25-27.

32. Oelke M, Bachmann A, Descazeaud A, et al; European Association of Urology. EAU guidelines on the treatment and follow-up of non-neurogenic male lower urinary tract symptoms including benign prostatic obstruction. Eur Urol. 2013;64(1):118-140.

33. Hvistendahl GM, Frøkiaer J, Nielsen S, Djurhuus JC. Gender differences in nighttime plasma arginine vasopressin and delayed compensatory urine output in the elderly population after desmopressin. JUrol. 2007; 178(6):2671-2676.

34. van Kerrebroeck P, Rezapour M, Cortesse A, Thüroff J, Riis A, Nørgaard JP. Desmopressin in the treatment of nocturia: a double-blind, placebo-controlled study. Eur Urol. 2007;52(1):221-229.

35. Mattiasson A, Abrams P, Van Kerrebroeck P, Walter S, Weiss J. Efficacy of desmopressin in the treatment of nocturia: a double-blind placebo-controlled study in men. BJU Int. 2002;89(9):855-862.
36. Lose G, Mattiasson A, Walter S, et al. Clinical experiences with desmopressin for long-term treatment of nocturia. J Urol. 2004;172(3): 1021-1025.

37. Kuo HC. Efficacy of desmopressin in treatment of refractory nocturia in patients older than 65 years. Urology. 2002;59(4):485-489.

38. Reynard JM, Cannon A, Yang Q, Abrams P. A novel therapy for nocturnal polyuria: a double-blind randomized trial of frusemide against placebo. Br J Urol. 1998;81(2):215-218.

39. Committee for Establishment of the Clinical Guidelines for Nocturia of the Neurogenic Bladder Society. Clinical guidelines for nocturia. Int J Urol. 2010;17(5):397-409.

40. Cho MC, $\mathrm{Ku}$ JH, Paick JS. Alpha-blocker plus diuretic combination therapy as second-line treatment for nocturia in men with LUTS: a pilot study. Urology. 2009;73(3):549-553.

41. Foley D, Ancoli-Israel S, Britz P, Walsh J. Sleep disturbances and chronic disease in older adults: results of the 2003 National Sleep Foundation Sleep in America Survey. J Psychosom Res. 2004;56(5):497-502.

42. Breyer BN, Shindel AW, Erickson BA, Blaschko SD, Steers WD, Rosen RC. The association of depression, anxiety and nocturia: a systematic review. J Urol. 2013;190(3):953-957.

43. Häkkinen JT, Shiri R, Koskimäki J, Tammela TL, Auvinen A, Hakama M. Depressive symptoms increase the incidence of nocturia: Tampere Aging Male Urologic Study (TAMUS). J Urol. 2008;179(5): 1897-1901.

44. Margel D, Shochat T, Getzler O, Livne PM, Pillar G. Continuous positive airway pressure reduces nocturia in patients with obstructive sleep apnea. Urology. 2006;67(5):974-977.

45. Addla SK, Adeyoju AB, Neilson D, O'Reilly P. Diclofenac for treatment of nocturia caused by nocturnal polyuria: a prospective, randomised, double-blind, placebo-controlled crossover study. Eur Urol. 2006;49(4): 720-725.

46. Kaye M. Nocturia: a blinded, randomized, parallel placebo-controlled self-study of the effect of 5 different sedatives and analgesics. Can Urol Assoc J. 2008;2(6):604-608.

47. Drake MJ, Mills IW, Noble JG. Melatonin pharmacotherapy for nocturia in men with benign prostatic enlargement. J Urol. 2004;171(3) 1199-1202.
Research and Reports in Urology

\section{Publish your work in this journal}

Research and Reports in Urology is an international, peer-reviewed, open access journal publishing original research, reports, editorials, reviews and commentaries on all aspects of adult and pediatric urology in the clinic and laboratory including the following topics: Pathology, pathophysiology of urological disease; Investigation and treatment of

\section{Dovepress}

urological disease; Pharmacology of drugs used for the treatment of urological disease. The manuscript management system is completely online and includes a very quick and fair peer-review system, which is all easy to use. Visit http://www.dovepress.com/testimonials.php to read real quotes from published authors. 